\title{
ECLETICA
}

www.scielo.br/eq

www.ecletica.iq.unesp.br

Volume 33, número 1, 2008

\section{Avaliação do desempenho analítico do método de determinação de TPH (Total Petroleum Hydrocarbon) por detecção no infravermelho}

\author{
A. R. Nascimento ${ }^{1}$, R.L. Ziolli ${ }^{*}$, J.T. Ararun, Jr', C. S. Pires ${ }^{l}$ e T.B. Silva ${ }^{2}$

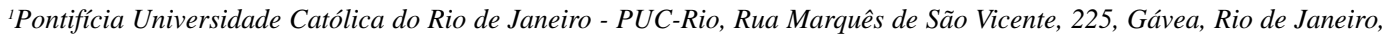 \\ RJ, 22453-900. \\ ${ }^{2}$ Universidade Estadual de Santa Cruz - UESC, Campus Soane Nazaré de Andrade, Rodovia Ilhéus-Itabuna, km 16, Ilhéus- \\ BA, Brasil. CEP: 45662-000 \\ *rlziolli@puc-rio.br
}

\begin{abstract}
Resumo: No presente trabalho os parâmetros de desempenho (validação intralaboratorial) da metodologia de determinação de TPH (Total Petroleum Hydrocarbons) foram determinados por detecção na região do infravermelho com o equipamento da Infracal TOG/TPH, visando aplicação em amostras de areia contaminadas com petróleo. Os ensaios foram realizados utilizando Óleo Marine Fuel 380, com densidade igual $0,987 \mathrm{~g} \mathrm{~cm}^{-3} \mathrm{e}$ viscosidade de $5313 \mathrm{cP} \mathrm{a} 20^{\circ} \mathrm{C}$. Este óleo foi fornecido pelo Centro de Pesquisa da Petrobrás (CENPES/PETROBRÁS/RJ), sendo o mesmo óleo derramado no acidente ocorrido em janeiro de 2000, na Baia de Guanabara, RJ, quando $1.300 \mathrm{~m}^{3}$ vazaram do duto que interliga a REDUC (Refinaria Duque de Caxias, RJ) ao terminal da Ilha d'Água/RJ, atingindo praias. Os resultados da validação indicaram que o desempenho da metodologia foi favorável à aplicação que se destina. Entre os parâmetros metrológicos obtidos neste trabalho, o limite de detecção do método foi de $4,06 \mathrm{mg} \mathrm{L}^{-1}$, consideravelmente inferior à faixa de concentração normalmente obtida para amostras em tais situações.
\end{abstract}

Palavras-chave: TPH; petróleo; areia contaminada; espectroscopia no infra-vermelho.

\section{Introdução}

Um dos parâmetros freqüentemente usado para a avaliação de uma contaminação proveniente de um vazamento de petróleo, é o "Total Petroleum Hydrocarbon", denominado TPH, que fornece informações sobre a concentração dos hidrocarbonetos totais presentes em determinada amostra [1].

A definição proposta para TPH, segundo a Agência de Proteção Ambiental dos Estados Unidos, USEPA, emprega dois conceitos. Um deles é o uso do fracionamento como uma alternativa para medir os constituintes da mistura de petróleo, dessa forma, tudo que for medido é somado e daí se tem o dado TPH. O outro conceito sugere que TPH são todos os hidrocarbonetos que foram extraídos de uma amostra e detectados por uma técnica particular [1,2].

Devido à existência de muitas substâncias químicas presentes no petróleo e seus derivados, não é prático medir cada uma dessas substâncias separadamente para efeito de monitoramento do progresso da remediação de uma área contaminada. Torna-se de maior utilidade medir a concentração total de hidrocarbonetos nessa área utilizando para efeitos de avaliação e monitoração o parâmetro TPH [1-6]. 
Além disso, como não há um único método estabelecido para sua determinação, diversas metodologias são empregadas com diferentes solventes de extração, distintas etapas de clean-up e diferentes técnicas analíticas de detecção (as técnicas freqüentemente usadas incluem a cromatografia gasosa, infravermelho, imunoensaio e gravimetria). Assim, uma mesma amostra pode produzir resultados diferentes de TPH, havendo, por isso, controvérsias no seu uso e aplicação e não sendo possível estabelecer uma correlação entre os resultados obtidos entre os diferentes métodos analíticos.

A interpretação dos resultados depende da compreensão das potencialidades e das limitações de cada técnica. Se usados indiscriminadamente, os dados de TPH podem ser enganadores e podem conduzir a uma avaliação inadequada.

Diante de tantas variações, a resposta de TPH torna-se limitada para estimar o risco à saúde humana e do ambiente, uma vez que amostras com as mesmas concentrações de TPH podem representar composições muito diferentes e, conseqüentemente, com efeitos toxicológicos distintos [7]. Por isso o parâmetro TPH não pode ser usado para estimar quantitativamente o risco à saúde. Se os dados de TPH indicarem que pode haver uma contaminação significativa do ambiente, outras análises devem ser realizadas de modo que o dano possa ser quantitativamente avaliado.

O TPH Criteria Working Group (1998) considera TPH como sendo um parâmetro útil e que pode ser usado para três principais finalidades: (i) identificação de uma contaminação; (ii) avaliação do grau de contaminação; e (iii) avaliação do progresso de uma remediação.

Entre as técnicas geralmente empregadas na avaliação de TPH destaca-se a Espectrometria de Infravermelho por Transformada de Fourier FT/IR. O constante emprego desta técnica criou muitas aplicações quantitativas para a espectrometria de infravermelho no monitoramento ambiental, no controle de qualidade e na análise de processos. Porém, devido a sua relativa complexidade, custo e exigências de acondicionamento, é inadequado mover um espectrômetro FT/IR para fora do laboratório para obtenção de medidas de campo [8 -11].
Para medidas de TPH no campo, são utilizados kits de diluição de amostra e instrumentos portáteis de infravermelho. Estes últimos apresentam algumas limitações, tais como a confiabilidade da faixa de trabalho, limite de detecção ou até mesmo a utilização do solvente adequado (Wilks Enterprise, 1995) [12]. Por isso, para a obtenção de dados preliminares num programa de monitoração no qual, geralmente o número de amostras é elevado, torna-se necessário o uso de um equipamento robusto, que possa gerar uma resposta com rapidez e com confiabilidade.

Este trabalho visa avaliar o desempenho de um método rápido e simples de medição, em campo, da contaminação de solos por hidrocarbonetos de petróleo. Para tanto, foram determinados alguns parâmetros de desempenho (validação intralaboratorial) da metodologia de determinação de TPH por detecção no infravermelho usando o infracal TOG/TPH, através da otimização do método estabelecido pela USEPA (USEPA Method 418.1) [2].

\section{Material e Métodos}

Para a determinação de TPH por IR foi utilizado o Analisador Infracal com cubeta de quartzo de caminho óptico igual a 10 milímetros, modelo CVH-50 da Wilks Enterprise [12\}, específico para a aplicação dos métodos 418.1 e 413.2 da EPA (Agência de Proteção Ambiental dos EUA) [2]. A metodologia usada neste trabalho seguiu o método USEPA 418.1 com modificações.

Para as amostras testes foram utilizadas amostras de solo areno-argiloso contaminado, por óleo cru, fornecidas pelo CENPES/Petrobrás. O solo contaminado foi adequadamente homogeneizado a temperatura ambiente utilizando um almofariz. Uma quantidade de 5,0 g da amostra homogeneizada foi pesada em balança analítica e extraída com $30 \mathrm{~mL}$ de tetracloroetileno (PCE), dentro de erlenmeyer com tampa de vidro, utilizando um agitador orbital a $160 \mathrm{rpm}$, por 4 horas, a temperatura ambiente. Após esse período, o sobrenadante foi filtrado utilizando um funil de vidro com um pedaço de algodão contendo $\mathrm{Na}_{2} \mathrm{SO}_{4}$ (sulfato de sódio). Posteriormente, a amostra foi novamente extraída com mais $15 \mathrm{~mL}$ 
de PCE por mais $30 \mathrm{~min}$, e filtrada. Os dois extratos foram então somados e avolumados com PCE em balão volumétrico a $50 \mathrm{~mL}$. Uma alíquota dessa solução foi então transferida para a cubeta e efetuada a leitura na região do Infravermelho, em aparelho TOG/TPH Infracal CV-50, previamente calibrado. Como padrão foi utilizada uma solução de Óleo Marine Fuel 380 (densidade do óleo igual $0,987 \mathrm{~g} \mathrm{~cm}^{-3}$ e viscosidade de $5313 \mathrm{cP}$ a $20^{\circ} \mathrm{C}$ ) em PCE. Este óleo foi fornecido pelo Centro de Pesquisa da Petrobras (CENPES), é o mesmo óleo do acidente de janeiro de 2000, quando $1300 \mathrm{~m}^{3}$ vazaram do duto que interliga a REDUC ao terminal da Ilha d'Água, no Rio de Janeiro, e atingiu as praias da Ilha de Paquetá. A partir da solução estoque do óleo em tetracloroetileno, com concentração de $10.000 \mathrm{mg} \mathrm{L}^{-1}$, foram feitas as diluições para a preparação das soluções padrão,com as concentrações variando na faixa de $10 \mathrm{mg} \mathrm{L}^{-1}$ a $10.000 \mathrm{mg} \mathrm{L}^{-1}$. A metodologia foi otimizada segundo recomendações descritas pelo INMETRO (2002).

\section{Resultados e discussão}

Otimização da metodologia de extração: solvente, massa de amostra e número de extrações

$\mathrm{O}$ solvente indicado pelo método USEPA 418.1 é o Freon-113 [2], porém, este solvente tem sido regulado pelas agências ambientais por ser nocivo ao meio ambiente. Vários solventes têm sido estudados para a substituição do Freon na determinação de TPH em amostras ambientais. A Tabela 1 apresenta algumas características dos solventes recomendados pela Wilks [12], para extração de TPH e aplicação nos equipamentos TOG/TPH.
O solvente que foi efetivamente utilizado nos experimentos foi o tetracloroetileno. Este é um bom substituto e, mesmo sendo medianamente tóxico, é amplamente usado como uma solução de limpeza e conseqüentemente pode ser aceitável para uso em laboratório, além de apresentar um custo inferior aos outros substitutos. Todavia, a pureza recomendada para o tetracloroetileno é de $99 \%$ e a pureza do solvente utilizado foi de $97,5 \%$, ou seja, abaixo da recomendada, devido a disponibilidade no mercado.

A Figura 1 mostra o espectro do solvente usado (tetracloroetileno) extraído da literatura.

Verifica-se que a região entre 3,23 a 3,51 $\mu \mathrm{m}\left( \pm 3095 \mathrm{~cm}^{-1}\right)$, que é a região de absorção da ligação $\mathrm{C}-\mathrm{H}$ e, portanto, a região de interesse para as determinações de TPH por IR, não apresenta interferência quando se usa o tetracloroetileno como solvente.

O método USEPA 418.1 original recomenda uma massa de amostra maior que a

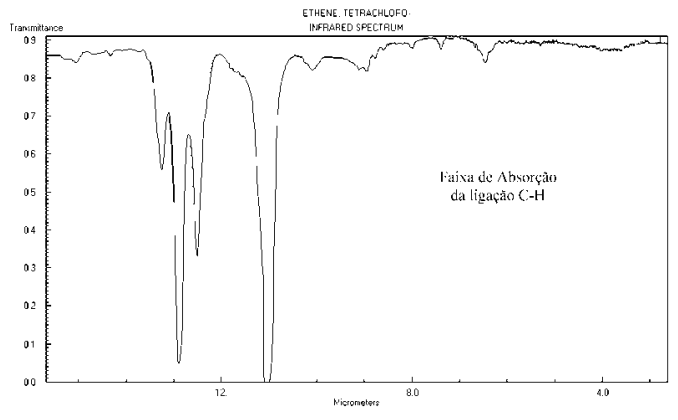

Figura 1. Espectro de absorção do Tetracloroetileno (C2C14). Fonte: NIST Chemistry WebBook (1985).

Tabela 1. Características dos solventes recomendados pela Wilks para extração de TPH (2002).

\begin{tabular}{|c|c|c|c|c|}
\hline & \multirow{2}{*}{\multicolumn{4}{|c|}{ Solventes }} \\
\hline & & & & \\
\hline & Freon-113 & Tetracloroetileno & Ak-225 & S-316 \\
\hline Pureza recomendada & $99+\%$ & $99+\%$ & $99+\%$ & N/A \\
\hline Eficiência de Extração & $91 \%$ & $87 \%$ & $88 \%$ & $98 \%$ \\
\hline $\begin{array}{lll}\text { Ponto de ebulição } & & \\
& & \text { C) }\end{array}$ & 48 & 121 & 54 & 134 \\
\hline Custo Médio & $\$ 205$ & $\$ 85$ & $\$ 140$ & $\$ 480$ \\
\hline \multirow{2}{*}{ Método de Extração } & USEPA & USEPA & USEPA & USEPA \\
\hline & $413.2 ; 418.1$ & $413.2 ; 418.1$ & $413.2 ; 418.1$ & $413.2 ; 418.1$ \\
\hline
\end{tabular}


usada neste trabalho $(5,0 \mathrm{~g})$ e apenas uma extração da amostra. Neste trabalho, a mesma amostra foi extraída duas vezes com menores porções de solvente, com o intuito de aumentar a eficiência da extração e minimizar a geração de resíduos. A primeira extração foi feita com 30 $\mathrm{mL}$ de solvente e a segunda com mais $15 \mathrm{~mL}$, completando o volume a $50 \mathrm{~mL}$.

Parâmetros de avaliação de desempenho da determinação de TPH utilizando o Infracal TOG/TPH

Faixa de trabalho para definição da faixa de concentração da curva analítica

Segundo a Wilks Enterprise [12], fabricante do equipamento utilizado nas determinações de TPH por IR, a faixa linear de trabalho do equipamento é de 0 a $10.000 \mathrm{mg}$ de TPH por litro de solução, conforme consta do manual do equipamento. Portanto, inicialmente essa foi a faixa de trabalho estudada para a definição das concentrações da faixa linear da curva analítica. A Tabela 2 mostra o valor médio da intensidade de sinal obtido para cada concentração, numa faixa de 0 a $10.000 \mathrm{mg} \mathrm{L}^{-1}$, obtido de três replicatas de cada concentração, e a respectiva variância. Os dados da tabela apresentam-se na Figura 2.

Tabela 2. Média dos valores de intensidade de sinal de três replicatas independentes em cada concentração da solução padrão.

\begin{tabular}{rcc}
\hline $\begin{array}{c}\text { Concentração } \\
\left(\mathrm{mg} \mathrm{L}^{-1}\right)\end{array}$ & $\begin{array}{c}\text { Intensidade } \\
\text { de sinal (ua*) }\end{array}$ & $\mu$ (variância) \\
\hline 0 & 1,1 & 0,7 \\
10 & 5,6 & 0,3 \\
50 & 27,4 & 1,3 \\
100 & 55,0 & 0,5 \\
200 & 101,8 & 0,2 \\
300 & 138,2 & 0,7 \\
400 & 168,0 & 1,5 \\
500 & 193,4 & 0,3 \\
1000 & 251,0 & 0,0 \\
2000 & 322,6 & 0,3 \\
3000 & 343,0 & 1,5 \\
4000 & 352,6 & 0,3 \\
5000 & 359,8 & 0,2 \\
10000 & 362,6 & 0,3 \\
\hline
\end{tabular}

*Unidades arbitrárias

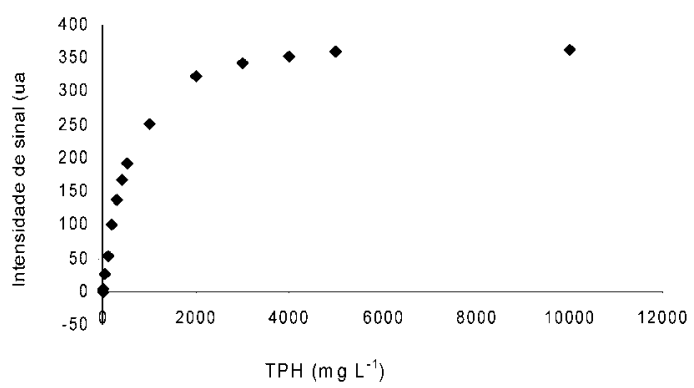

Figura 2. Curva analítica na faixa de trabalho sugerida pelo fabricante do equipamento.

A ampla faixa de trabalho sugerida pelo fabricante, indica uma grande versatilidade no uso do Infracal nas determinações de TPH nas amostras de solos contaminados com petróleo, onde as concentrações chegam até $50.000 \mathrm{mg} \mathrm{kg}^{-1}$.

\section{Linearidade da curva analítica}

$\mathrm{Na}$ Figura 3, pode-se observar que não existe uma relação linear em toda a faixa de trabalho especificada pelo fabricante do equipamento. A linearidade pode ser melhor observada na faixa de concentração que vai de 0 a $500 \mathrm{mg}$ $\mathrm{L}^{-1}$ (Figura 3), sendo essa a faixa determinada para a curva de analítica.

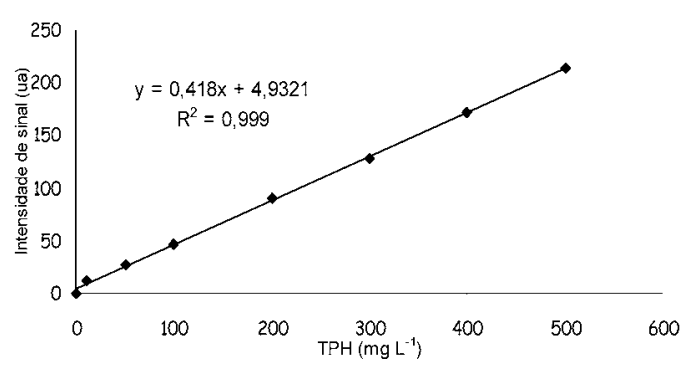

Figura 3. Faixa linear da curva analítica.

Após a otimização do método, obteve-se um coeficiente de correlação linear de $\mathrm{R}^{2}=0,999$, que mostra uma ótima correlação entre os dados obtidos.

Protocolo de validação da curva de calibração de 0 a $500 \mathrm{mg} \mathrm{L}^{-1}$

Sensibilidade

Segundo INMETRO (2000) [13], a sensi-

Ecl. Quím., São Paulo, 33(1): 35-42, 2008 
bilidade é um parâmetro que demonstra a variação da resposta em função da concentração do analito. Pode ser expressa pela inclinação da curva de regressão linear de calibração, conforme a equação abaixo e é determinada simultaneamente aos testes de linearidade. Assim, pode-se observar a sensibilidade do método pela inclinação (coeficiente angular) da curva de regressão linear da calibração, conforme representado na equação 1 .

$S=\mathrm{dx} / \mathrm{dc}$

(equação 1)

Onde: $S=$ sensibilidade;

$d \mathrm{x}=$ variação da resposta;

$d c=$ variação da concentração.

Assim, através da curva analítica apresentada na Figura 3, obtém-se o valor 0,466 abs L $\mathrm{mg}^{-1}$ para a sensibilidade do método na faixa de 0 a $500 \mathrm{mg} \mathrm{L}^{-1}$.

Embora a faixa de trabalho obtida pelo equipamento seja ampla, optou-se fazer a calibração apenas na faixa linear da curva analítica, onde é maior a variação da resposta do instrumento de medição, obtendo-se melhor sensibilidade do método analítico. Além disso, se a curva de calibração não é linear, o valor da sensibilidade varia com a concentração do analito, enquanto trabalhando na faixa linear, o valor da sensibilidade fica constante em toda a faixa de trabalho.

\section{Limite de detecção}

Há controvérsias em relação ao termo "limite de detecção", apesar de ser usado em alguns documentos. O limite de detecção do equipamento $(L D)$ é definido como a concentração do analito que produz um sinal de três a cinco vezes a razão ruído/sinal do equipamento. O limite de detecção do método $(L D M)$ é definido como a concentração mínima de uma substância medida e declarada com 95\% ou $99 \%$ de confiança de que a concentração do analito é maior que zero. O LDM é determinado através de análise completa de uma dada matriz contendo o analito. Para a validação de um método analítico, é normalmente suficiente fornecer uma indicação do nível em que a detecção do analito começa a ficar problemática, ou seja, "branco + $3 s " e$ " $0+3 s$ “, considerando análise de sete ou mais amostras de branco e de brancos com adição, respectivamente [13-20].
Os valores obtidos para a média das intensidades de sinal obtidas para 10 leituras do branco para o cálculo do limite de detecção foi $0,11 \pm$ 0,08 . Os cálculos para a determinação do LD e LDM, utilizando o valor de $t$ unilateral $(3,143)$, para $99 \%$ de confiança e admitindo uma distribuição normal dos resultados, estão representados na equação 2 e 3 , respectivamente.

$\mathrm{LD}=\bar{X}+\mathrm{ts}$

(equação 2)

$\mathrm{LDM}=\mathrm{ts}$

Onde: $\mathrm{LD}=$ limite de detecção;

LDM = limite de detecção do método;

$X=$ média das intensidades de sinal obtidas para $\mathrm{N}$ leituras do branco;

$s=$ desvio padrão da média das leituras do branco.

Assim, foram obtidos os valores de 0,361 abs para o LD e 0,251 abs para o LDM. Em termos de concentração, o limite de detecção é 4,29 $\mathrm{mg} \mathrm{L}^{-1}$ e o limite de detecção do método é $4,06 \mathrm{mg} \mathrm{L}^{-1}$. Embora o valor de LDM obtido seja alto em comparação com outras técnicas analíticas para a determinação de TPH, o resultado pode ser considerado satisfatório para o fim a que se destina visto que os valores de concentração usualmente obtidos nas análises das amostras de areia após um derramamento de óleo normalmente são muito superiores ao LDM. Além disso, é necessário considerar que as determinações foram realizadas num equipamento portátil para aplicação em trabalho de campo.

\section{Limite de quantificação}

O Limite de Quantificação é a menor concentração do analito que pode ser determinada com um nível aceitável de precisão e veracidade. Considerou-se aqui como sendo a concentração do analito correspondente ao valor da média do branco mais 5 desvios padrão, estão representados na equação 4 [13-20].

$$
\mathrm{LQ}=\bar{X}+5 \mathrm{~s} \quad \text { (equação 4) }
$$

Onde: $\mathrm{LQ}=$ limite de quantificação; $X=$ média das intensidades de sinal obtidas para $\mathrm{N}$ leituras do branco; $s=$ desvio padrão da média das leituras do branco. 
Assim, o valor do LQ foi de 0,510 abs sendo que, em termos de concentração, o limite de quantificação é 4,61 $\mathrm{mg} \mathrm{L}^{-1}$.

\section{Exatidão}

Exatidão do método é definida como sendo a concordância entre o resultado de um ensaio e o valor de referência aceito como convencionalmente verdadeiro. A exatidão, quando aplicada a uma série de resultados de ensaio, implica numa combinação de componentes de erros aleatórios e sistemáticos (tendência). Os processos normalmente utilizados para avaliar a exatidão de um método são, entre outros: uso de materiais de referência, participação em comparações interlaboratoriais e realização de ensaios de recuperação [13-20].

Calculou-se o erro como sendo a diferença entre o resultado de um ensaio e o resultado aceito como referência (concentração teórica). Uma forma de avaliar a exatidão do método é através do cálculo do erro relativo (ER), expresso em percentagem.

Devido à impossibilidade de se fazer os ensaios com material de referência, teve-se que usar soluções preparadas dentro das condições do laboratório. Assim, preparou-se uma solução padrão com concentração de TPH calculada de $102,88 \mathrm{mg} \mathrm{L}^{1}$, concentração teórica usada como valor de referência, e efetuou-se 10 leituras desta solução. A concentração de TPH média obtida e o respectivo desvio padrão foram de $122,51 \mathrm{mg} \mathrm{L}^{-}$ ${ }^{1} \pm 4,32 \mathrm{mg} \mathrm{L}^{-1}$ o que produz um erro relativo de 19\%. Enfatiza-se aqui que a aplicação da metodologia visa aplicação em programas preliminares de monitoramento nos quais usualmente há um número elevados de amostras para análise em curto espaço de tempo. Nesses casos, geralmente a obtenção de valores que indiquem a presença ou não de contaminação e sua dimensão (a ordem de grandeza) podem ser suficiente.

\section{Precisão}

\section{Repetitividade}

Repetitividade é o grau de concordância entre os resultados de medições sucessivas de um mesmo mensurando, efetuadas sob as mesmas condições de medição [14]. A repetitividade pode ser expressa quantitativamente em termos da característica de dispersão dos resultados e pode ser determinada por meio da análise de padrões. Para a determinação da repetitividade nesse trabalho foi utilizada solução padrão na concentração de $300 \mathrm{mg} \mathrm{L}^{-1}$ de TPH. A repetitividade é expressa pelo desvio padrão de 10 leituras que neste trabalho foi de 2,08 .

A partir do desvio padrão dos resultados dos ensaios sob condição de repetitividade é aconselhável calcular o limite de repetitividade " $r$ " que capacita o analista a decidir se a diferença entre análises duplicatas de uma amostra, determinada sob condições de repetitividade, é significante. Para um nível de confiança de 95\%, o limite de repetitividade ( $r$ é avaliado segundo equação 5.

$$
\mathrm{r}=2,8 . \mathrm{Sr}
$$$$
\text { (equação 5) }
$$

Onde: $\quad \mathrm{Sr}=$ desvio-padrão de repetitividade

O limite de repetitividade (r) para esta determinação é de 5,82 abs, calculado num nível de confiança de 95\% segundo INMETRO 2000.

\section{Reprodutibilidade (Precisão intermediária)}

É o grau de concordância entre os resultados das medições de um mesmo mensurando, efetuadas sob condições variadas de medição (VIM). Embora a reprodutibilidade não seja um componente de validação de método executado por um único laboratório, é considerada importante quando um laboratório busca a verificação do desempenho dos seus métodos em relação aos dados de validação obtidos através de comparação interlaboratorial. A precisão sob condições de reprodutibilidade é obtida, por exemplo, de resultados dos ensaios com o mesmo método, variando-se laboratórios, operadores ou equipamentos.

Nesse trabalho a precisão sob condições de reprodutibilidade foi obtida por analistas diferentes e usando uma amostra de areia contaminada com petróleo numa concentração aproximada de $5.000 \mathrm{mg} \mathrm{kg}^{-1}$ de TPH. Foi calculado o desvio padrão para a variável TPH, sendo adotado a média como resultado. O desvio padrão foi de $124,05 \mathrm{mg} \mathrm{kg}^{-1}$.

A reprodutibilidade é expressa pelo desvio 
padrão obtido sob condições de reprodutibilidade associada aos resultados. A partir do desvio padrão obtido sob condições de reprodutibilidade foi possível calcular a variância de reprodutibilidade $\left(\mathrm{S}_{\mathrm{R}}{ }^{2}\right)$ associada aos resultados, e o limite de reprodutibilidade "R" conforme equação 6 [13-15].

$$
\begin{array}{ll}
\mathrm{R}=2,8 \sqrt{\mathrm{S}_{\mathrm{R}}^{2}} \quad \text { (equação 6) } \\
\mathrm{R}=347,34
\end{array}
$$

O limite de reprodutibilidade $(\mathrm{R})$ para esta determinação é de $347,34 \mathrm{mg} \mathrm{kg}^{-1}$, calculado num nível de confiança de $95 \%$ segundo INMETRO 2000.

\section{Conclusões}

No presente trabalho foi otimizada uma metodologia analítica simples e de baixo custo para a determinação de TPH em solos contaminados com petróleo e derivados. $\mathrm{O}$ enfoque princi- pal deste estudo não foi a quantificação dos compostos individuais e sim desenvolver uma metodologia capaz de indicar a presença ou ausência de contaminação por petróleo. Assim, para fins de monitoramento ambiental preliminar, esta técnica é indicada por ser processo de análise mais rápido e econômico se comparado a outros processos usualmente utilizado para a determinação de TPH.

A faixa de trabalho sugerida pelo fabricante do equipamento utilizado não apresentou linearidade em todas as concentrações. A faixa linear obtida experimentalmente neste trabalho foi de 0 a $500 \mathrm{mg} \mathrm{L}^{-1}$ e os resultados obtidos para o limite de detecção e o de quantificação indicaram uma aplicação favorável ao uso a que se destina. Assim, pode-se concluir que o desempenho do método de determinação de TPH em areia por detecção no infravermelho foi adequado.

Recebido em 30 de outubro de 2007

Aceito em 27 de fevereiro de 2009

A. R Nascimento, R. L.Ziolli, J. T. Araruna, Jr, C. S. Pires, T.B. Silva. Validation of method for the determination of TPH (Total Petroleum Hydrocarbon) by infrared detection.

\begin{abstract}
This work deals with an optimization of TPH (Total Petroleum Hydrocarbon) analysis methodology for samples of contaminated sands, validating the infrared detection technique through the use of Infracal TOG/TPH equipment. Tests were validated using Marine Fuel 380 oil, density 0.987 $\mathrm{g} \mathrm{cm}^{-3}$ and viscosity $5313 \mathrm{cP}$ at $20^{\circ} \mathrm{C}$. This oil sample was kindly supplied by Petrobras Research Centre (CENPES), and is the same oil that leaked from a pipeline in REDUC Refinery on January 2000, contaminating several beaches in Guanabara Bay, including Anil and Mauá. The validation results suggested that the methodology performance was adequate for this application. Amongst the metrological parameters obtained from this work, the detection limit, $4.06 \mathrm{mg} \mathrm{L}^{-1}$, was a plus; since it was far below to the concentration range obtained from this samples
\end{abstract}

Keywords: TPH; petroleum; sand contamination; infra-red spectroscopy. 


\section{Referências}

[1] TPH Criteria Working Group. Analysis of Petroleum Hydrocarbons in Envirronmental Media, 1998. Disponível em: <http://www.aehs.com >.

[2] U.S. EPA Method 418.1. Petroleum hydrocarbons. Total recoverable (spectrophotometric, infrared), issued 1978. Methods for Chemical Analysis of Water and Wastes. 3rd ed. Cincinnati, OH: U.S. Environmental Protection Agency, 1983.

[3] M. Gerhard, S. Wolfgang, K. Jürgen. Field Method for the Detection of Total Petroleum Hydrocarbon in Water and Soil Samples. Presented on the "Fifth International Symposium on Field Analytical Methods for Hazardous Wastes and Toxic Chemicals", Las Vegas, NV, USA, Jan. 29-31, (1997).

[4] P. A. Kulakow, L. A. Erickson, A Nationwide Field Test of Petroleum-Contaminated Soils. Department of Agronomy, Throckmorton Hall, Kansas State University, Manhattan, KS And Department of Chemical Engineering Durland Hall, Kansas State University, Manhattan, KS. March 2000.

[5] P. Lambert, M. Fingas. A critical review of field total petroleum hydrocarbon (TPH) analysis, Part II, in: Proceedings of the Twentieth Arctic and Marine Oilspill Program, Technical Seminar, Environment Canada, Ottawa, Ont., (1997) 175.

[6] P. Lambert, M. Fingas, M. Goldthorp, 2001. Journal of Hazardous Materials 83 (2001) 65.

[7] W. H. Weisman, J. Soil Contamination. 7(1) (1998) 15.

[8] M. J. Kennish, Pratical handbook of estuarine and marine pollution. CRC Press - Marine Science Series, New York, 1997.

[9] C. Robert, T. David, Journal of Chromatography A, 785 (1997) 269.
[10] C. S. Frick, Analytical Techniques for Soluble Component Analysis. IN: Proceedings, Sampling and Analytical Methods for Determining Petroleum Hydrocarbons in Groundwater and Soil. Amer. Petro. Inst., Wash. D.C, 1987.

[11] T. L. Potter, Analysis of Petroleum Contaminated Soil and Water: An Overview. IN: Petroleum. Contaminated Soils, Vol. 2, Lewis Publications, Inc. Chelsea, MI, 1989.

[12] Wilks Enterprise, 1995. InfraCal® TOG/TPH, Analyzer, Cuvette Model CVH, User's Guide. Disponível em: < http://www.wilksir.com/pdf/user_guide_model_cvh.pdf >.

[13] INMETRO. Orientação sobre Validação de Métdos de Ensaios Químicos. Duque de Caxias, INMETRO 2002.

[14] VIM - Vocabulário Internacional de Termos Fundamentais e Gerais de Metrologia, 1995.

[15] Eurachem. The Fitness for Purpose of Analytical Methods: A Laboratory Guide to Method Validation and Related Topics. Teddington, Eurachem, 1998.

[16] INMETRO \& ABPTI. Guia de Implantação da Confiabilidade Metrológica. Rio de Janeiro, 1986.

[17] INMETRO. Guia Para Laboratórios Químicos: Um Auxílio à Organização e ao Credenciamento. Rio de Janeiro, Interciência, 2000.

[18] INMETRO. Vocabulário Internacional de Termos Fundamentais e Gerais de Metrologia. Duque de Caxias, INMETRO 1995.

[19] E. P. Cerqueira Neto, E. P. Gerenciando a Qualidade Metrológica. Rio de Janeiro, Imagem, 1993.

[20] F. J. Rosenberg, A. B. M. Silva, Sistemas da qualidade em laboratórios de Ensaio: Guia Prático para a Interpretação e Implementação da ABNT ISO/IEC Guia 25. Rio de Janeiro, Quality Mark, 2000. 